\title{
Optimal and Robust Walking using Intrinsic Properties of a Series-Elastic Robot
}

\author{
Alexander Werner ${ }^{*}$, Bernd Henze* ${ }^{*}$ Florian Loeff ${ }^{*}$, Sigrid Leyendecker ${ }^{\dagger}$ and Christian Ott ${ }^{*}$
}

\begin{abstract}
Series-Elastic Actuators (SEA) have been proposed as a technology to build robust humanoid robots. The aim of this work is to generate efficient and robust walking for such robots. We present a combined approach which exploits the system dynamics through optimization based trajectory generation and a robust control scheme. The compliant actuator dynamics are explicitly modeled in the optimal control problem. For local stabilization, a passivity based tracking controller distributes the required control forces onto the available contacts. Additionally, a predictive control scheme for step adaptation is presented, which provides feasible contact points in the future. Using a reduced model, this combines efficient walking with robustness against model or environment uncertainties and external disturbances.
\end{abstract}

\section{INTRODUCTION}

Electrically actuated legged robots are usually operated in a very careful manner as abrupt external forces generated by the dynamics of the locomotion process itself can damage the robot. In order to overcome this limitation, series elastic actuators decouple the motor via a spring from the link-side motion. The resulting fourth order dynamics can be used to efficiently generate locomotion patterns. This is most effective if the intrinsic system behavior is used. Suitable trajectories can be generated by analyzing Eigenmodes of the system [1] or by application of numerical methods. In this work we combine an optimization based trajectory generation with a robust control framework.

On a SEA robot, not every dynamic link motion can be generated due to actuator limitations. This depends essentially on the combination of the motor and link side inertia and the limits on the actuator itself. Some state-of-the-art robots reduce this problem by having their actuators concentrated in the trunk and hence have very light weight links. This also allows the use of low dimensional dynamic models with little error. However, when developing a full-body universal humanoid robot, at least some part of the actuators will be located in the lower extremities, which motivates the use of models with distributed mass. For these models, numerical trajectory optimization is the established method for finding

\footnotetext{
${ }^{*}$ With the Institute for Robotics and Mechatronics, German Aerospace Center (DLR). ${ }^{\dagger}$ With the Department of Mechanical Engineering, University of Erlangen-Nuremberg.

This work was partially supported by the Helmholtz Association (VH-NG-808) and by the European Commission (H2020-ICT645097 COMANOID).
}

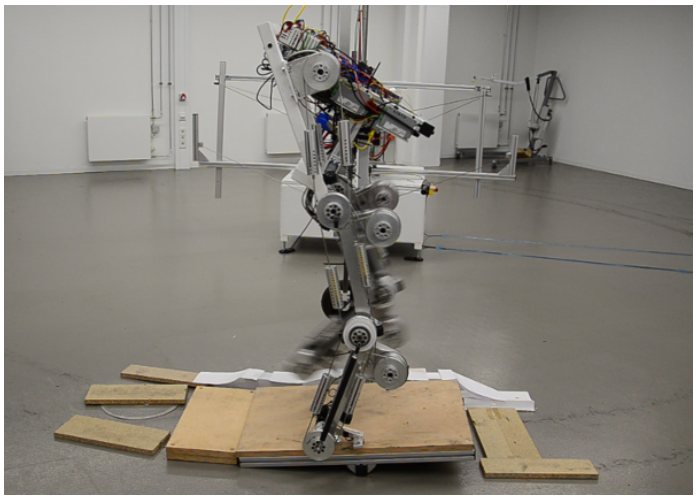

Fig. 1. Robot dynamically walking over an unmodelled see-saw like obstacle.

feasible and optimal locomotion trajectories. In order to benefit from the elastic actuation and to guarantee the feasibility of the motions, this work is based on our previously presented approach for trajectory generation for cyclic walking patterns for elastic systems [2].

Using optimal trajectories alone will not lead to robust walking due to uncertainties in the robot and environment model. Therefore, we use a tracking controller which utilizes the currently active contacts to stabilize the trajectory, which we previously presented in [3]. The controller was evaluated for walking in [4] for simulated rigid body robots. In this work, we present the usage of this controller on a SEA robot and investigate stiffness design considering the intrinsic system properties. Special care is taken to minimize the control effort required to stabilize the system by keeping stiffness shaping at a minimum. Second, we propose a step adaptation scheme using predictive control that uses the center of mass (CoM) dynamics and contact force constraints to compute future steps. Based on these results, the next whole-body trajectory is chosen and commanded to the tracking controller.

For the experimental part, we use the series-elastic biped robot C-Runner which was recently presented in [5]. Experiments on flat floor show the general feasibility and performance of the method, including an analysis of the cost of transport. Walking over obstacles as shown in Fig. 1 demonstrates the robustness.

This paper is structured as follows: First, we review other robust walking robots with similar technologies in Section II followed by the problem statement summarizing the task at hand in Section III. The trajectory generation of cyclic walking primitives is presented in 


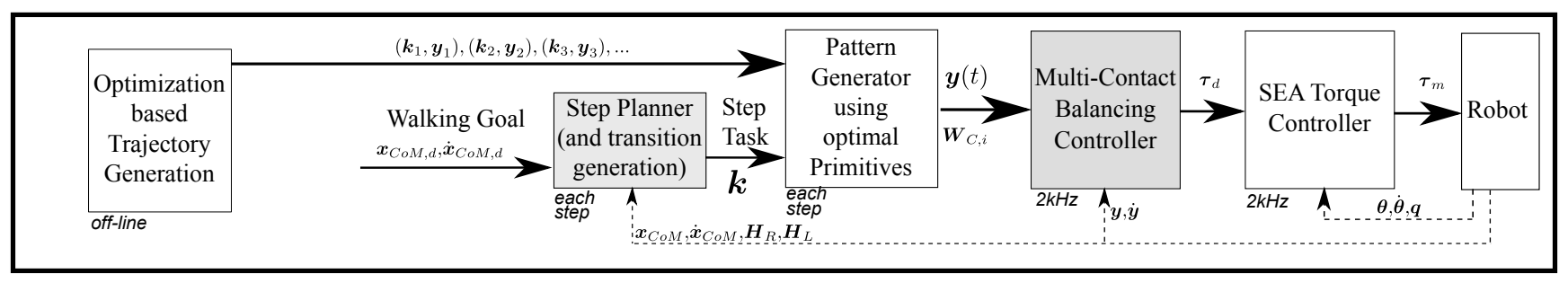

Fig. 2. Overview of the approach to generate optimal and robust walking.

Section IV. Afterwards the step planning with feasible contact forces is discussed in Section V. Section VI presents a short recapitulation of the balancing controller and addresses the stiffness computation in detail. Section VII documents the SEA torque controller, which is used to generate the torque demanded by the multicontact tracking controller. The results from the experiments are discussed in Section VIII and Section IX while Section X summarizes the work.

\section{RELATED WORK}

The significance of mechanically robust and efficient locomotion was recognized early in [6]. The first SEA concept robots, like M2V2, demonstrated step adaptation to maintain balance [7]. Complementary to that, COMAN displayed ZMP based walking, but with high immunity to disturbances [8]. Both robots featured flat feet, in contrast to the works on ATRIAS (especially MARLO and conceptually related robot Cassie), which imitate the SLIP model. Those robots were built with very light-weight legs and point feet and are thus always underactuated in single support [9], [10]. Another SEA robot is MABEL [11] which has shown high performance walking and running.

Limit cycle walking with underactuated mechanisms was studied from a control oriented perspective by means of the hybrid zero dynamics approach in [12], [13]. In this concept, a set of virtual constraints is used in order to parameterize the robot configuration throughout a step. The shape of the virtual constraints is determined via optimization such that the remaining hybrid zero dynamics under the effect of the impacts at touch-down of swing leg admits a stable limit cycle. Extensions of this concept include walking in 3D [14] as well as walking with feet [13]. Moreover, the extension to compliant robots has been presented in [15], where improved energetic efficiency due to the compliance is reported.

Recently DURUS combined the efficiency of passive elastic elements above the feet with rigid actuation in all other directions. For maximum energy efficiency this descision is understandable, but the general robustness of a robot actuated with SEA in all joints is lost. This robot is e.g. not robust against falling on the knees. The Hybrid Zero Dynamics concept was used here to provide trajectories and the control approach. This led to human like gait with heel and toe phases in [16].

\section{Problem Statement}

This paper addresses the problem of generation optimal and robust walking trajectories for a soft-elastic, planar, bipedal robot with distributed masses. The concept is general and the aspects that can also be applied to a rigid robot have been evaluated on a full 3D humanoid [3], [17].

The approach is summarized in Fig. 2. A set of trajectories are generated off-line by solving an optimal control problem. During execution the SEA torque controller abstracts the robot as a torque controlled robot. The multi-contact balancing controller provides stabilization of the trajectory using the feet currently in contact. A continuous walking trajectory is assembled by the pattern generator using step tasks produced by the step planner. The stepping tasks are provided on-line using the model predictive control based step planner. The step planner is executed on touchdown of the swing foot and computes which step should be taken next.

The configuration of the robot is described by

$$
\boldsymbol{y}=\left[\begin{array}{l}
\boldsymbol{x} \\
\boldsymbol{q}
\end{array}\right] \in \mathbb{R}^{9}
$$

with $\boldsymbol{x} \in \mathbb{R}^{3}$ representing the base coordinates, containing the horizontal translation, vertical translation and base link rotation $\varphi$, and $\boldsymbol{q} \in \mathbb{R}^{6}$ the joint angles. The model dimensions match the robot used for the experiments. Fig. 3 illustrates the coordinates in detail.

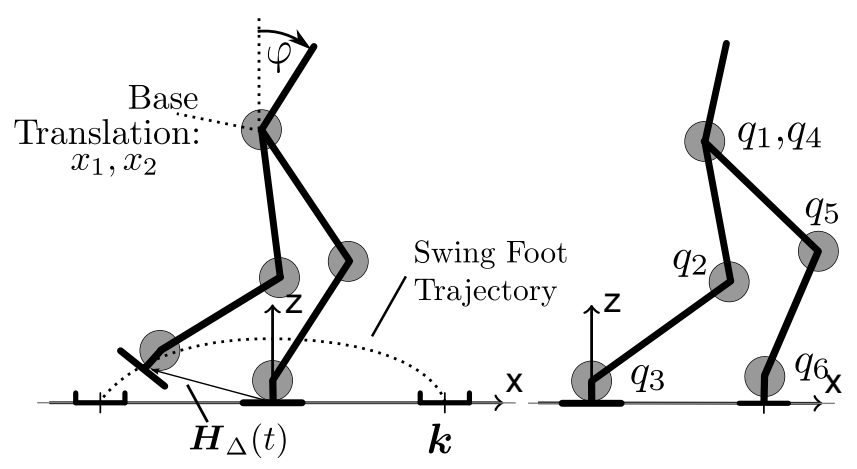

Fig. 3. Kinematic of the planar robot. Left: Schematic motion of the swing foot during the single-support phase for the step task $\boldsymbol{k}$. Right: Closed kinematic Loop during the double-support phase.

The link side dynamics of the robot addressed in this 
paper is described by

$$
\boldsymbol{M}(\boldsymbol{y}) \ddot{\boldsymbol{y}}+\boldsymbol{C}(\boldsymbol{y}, \dot{\boldsymbol{y}}) \dot{\boldsymbol{y}}+\boldsymbol{g}(\boldsymbol{y})=\left[\begin{array}{l}
\mathbf{0} \\
\boldsymbol{\tau}
\end{array}\right]+\sum_{i=0}^{N_{\mathrm{C}}} \boldsymbol{J}_{\mathrm{C}, i}^{\mathrm{T}} \boldsymbol{W}_{\mathrm{C}, i}
$$

with the inertia matrix $\boldsymbol{M} \in \mathbb{R}^{9 \times 9}$, Coriolis matrix $\boldsymbol{C} \in$ $\mathbb{R}^{9 \times 9}$, gravity terms $\boldsymbol{g} \in \mathbb{R}^{9}$, joint torques $\boldsymbol{\tau} \in \mathbb{R}^{6}$, contact Jacobians $\boldsymbol{J}_{\mathrm{C}, i} \in \mathbb{R}^{3 \times 9}$, and contact wrenches $\boldsymbol{W}_{\mathrm{C}, i} \in$ $\mathbb{R}^{3}$ with the index $i$ to distinguish between contacts. For the sake of clarity, the dependencies will be omitted for the remainder of the paper. The walking gait is defined by two phases, the single support phase $(S S P)$ and the double support phase $(D S P)$, which causes the number of contacts $N_{C}$ to switch between $N_{\mathrm{C}}=1$ and $N_{\mathrm{C}}=2$. The motor dynamics are coupled to the link side dynamics through linear springs forming an SEA:

$$
\begin{aligned}
\boldsymbol{\tau} & =\boldsymbol{K}(\boldsymbol{\theta}-\boldsymbol{q}) \\
\boldsymbol{B} \ddot{\boldsymbol{\theta}} & =\boldsymbol{\tau}_{m}-\boldsymbol{\tau}
\end{aligned}
$$

where $\boldsymbol{K} \in \mathbb{R}^{6 \times 6}$ is the stiffness matrix, $\boldsymbol{\theta} \in \mathbb{R}^{6}$ the motor positions, $\boldsymbol{B} \in \mathbb{R}^{6 \times 6}$ the projected motor inertia matrix, and $\boldsymbol{\tau}_{m} \in \mathbb{R}^{6}$ the motor torques.

In order to achieve efficient locomotion, a motor torque cost function is used for the planning as approximation for the electric cost of transport:

$$
\Gamma=\int_{0}^{t_{f}} \boldsymbol{\tau}_{m}^{T} \boldsymbol{\tau}_{m} \mathrm{~d} t
$$

with the trajectory duration $t_{f}$. A feasible motion is ensured by satisfying the contact force constraints

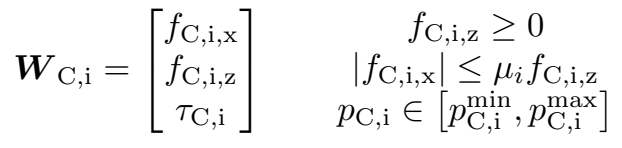

with the tangential foot forces $f_{\mathrm{C}, \mathrm{i}, \mathrm{x}}$ the normal foot forces $f_{\mathrm{C}, \mathrm{i}, \mathrm{Z}}$, foot torques $\tau_{\mathrm{C}, \mathrm{i}}$, and the center of pressure $(\mathrm{CoP}) p_{\mathrm{C}, \mathrm{i}}=\tau_{\mathrm{C}, \mathrm{i}} / f_{\mathrm{C}, \mathrm{i}, \mathrm{z}}$ limited by the foot geometry $p_{\mathrm{C}, \mathrm{i}}^{\min }, p_{\mathrm{C}, \mathrm{i}}^{\max }$.

On kinematic level, link position limits and environment collision constraints have to be ensured. For the actuator side, the motor speed and torque are constrained by

$$
|\dot{\boldsymbol{\theta}}| \leq \dot{\boldsymbol{\theta}}_{\max } \quad \text { and } \quad\left|\boldsymbol{\tau}_{m}\right| \leq \boldsymbol{\tau}_{\operatorname{m,max}}
$$

Given a series of cyclic gait primitives, which alternate between SSP and DSP on both legs, a continuous trajectory can be provided. In order to stabilize this trajectory with minimal control effort, the intrinsic system stiffness $\boldsymbol{K}$ should be used to compute the compliance parameters for the tracking controller (see Sec. VI).

As the stability region of a pure balancing control approach is small, future step positions have to be adapted to extend the region (see Sec. V).

\section{Trajectory Generation}

Providing trajectories which take into account all dynamic effects of a SEA walking robot is currently only possible off-line due to the high computational effort required. In this work, we use the optimal control problem formulation presented in [2] to generate cyclic walking primitives. This formulation explicitly models the actuator dynamics.

The robot can be completely parameterized by $\boldsymbol{q}$ if we assume that one foot is always in contact with the ground during the gait. Thus, the base state can be computed using the contact constraint of dimension 3

$$
\boldsymbol{H}_{\mathrm{STANCE}}=\boldsymbol{I}
$$

where $\boldsymbol{H}_{\mathrm{STANCE}}$ is the homogeneous transformation matrix of the position of the stance foot, $\boldsymbol{I}$ the identity matrix [17]. In order to provide higher derivatives of the state $\boldsymbol{y}$, equivalent velocity level constraints are required. However, in order to be independent of this restriction, the complete state $\boldsymbol{y}$ is used to express the equations of motion (2). The solution of inverse dynamics is uniquely defined, given the states $\boldsymbol{y}, \dot{\boldsymbol{y}}$ and $\ddot{\boldsymbol{y}}$, and thus are $\boldsymbol{\tau}$ and $\boldsymbol{W}_{C, i}$ but only in single support (2). To compute $\boldsymbol{\tau}$ and $\boldsymbol{W}_{C, i}$ during the double support phase, the redundancy of the right-hand side must be resolved:

$\boldsymbol{O}(\boldsymbol{y}, \dot{\boldsymbol{y}}, \ddot{\boldsymbol{y}})=\left[\begin{array}{c}\mathbf{0} \\ \boldsymbol{\tau}\end{array}\right]+\left[\begin{array}{ll}\boldsymbol{\alpha}(t) \boldsymbol{J}_{\mathrm{C}, 1}^{T} & (1-\boldsymbol{\alpha}(t)) \boldsymbol{J}_{\mathrm{C}, 2}^{T}\end{array}\right]\left[\begin{array}{l}\boldsymbol{W}_{\mathrm{C}, 1} \\ \boldsymbol{W}_{\mathrm{C}, 2}\end{array}\right]$

which is achieved using the given weights $\boldsymbol{\alpha}$ [2], and then solving the equation system using a least-square solver. Note, that the left-hand side of (2) is shortened for simplicity to $\boldsymbol{O}(\boldsymbol{y}, \dot{\boldsymbol{y}}, \ddot{\boldsymbol{y}})$.

To compute $\boldsymbol{\tau}_{m},(3)$ is differentiated twice and combined with (4) into:

$$
\ddot{\boldsymbol{\tau}}=\boldsymbol{K}(\ddot{\boldsymbol{\theta}}-\ddot{\boldsymbol{q}})=\boldsymbol{K}\left(\boldsymbol{B}^{-1}\left(\boldsymbol{\tau}_{m}-\boldsymbol{\tau}\right)-\ddot{\boldsymbol{q}}\right) .
$$

The information on $\ddot{\tau}$ can be provided by differentiating (2) twice:

$$
\begin{gathered}
\ddot{\boldsymbol{O}}(\boldsymbol{y}, \dot{\boldsymbol{y}}, \ddot{\boldsymbol{y}}, \dddot{\boldsymbol{y}}, \dddot{\boldsymbol{y}})=\left[\begin{array}{l}
\mathbf{0} \\
\boldsymbol{\tau}
\end{array}\right]+\ldots \\
\ldots \quad+\sum_{i=0}^{N_{C}}\left[\ddot{\boldsymbol{J}}_{C, i} \boldsymbol{W}_{C, i}+2 \dot{\boldsymbol{J}}_{C, i} \dot{\boldsymbol{W}}_{C, i}+\boldsymbol{J}_{C, i} \ddot{\boldsymbol{W}}_{C, i}\right]
\end{gathered}
$$

and removing any existing redundancy using (9). This allows us to minimize the cost function (5) w.r.t. the inequality constraints mentioned in Sec. III. Additionally, the kinematics of each step is enforced by using the following equality constraints of dimension 3

$$
\begin{aligned}
& \boldsymbol{H}_{\Delta}(0) \stackrel{!}{=} \boldsymbol{H}_{\text {start }} \\
& \boldsymbol{H}_{\Delta}(t) \stackrel{!}{=} \boldsymbol{H}_{D S P} \quad \forall t \in\left[t_{s w}, t_{f}\right]
\end{aligned}
$$

on the position of the swing foot $\boldsymbol{H}_{\Delta}(t)$ during the double support phase defined by the interval $\left[t_{s w}, t_{f}\right]$. which fully define the cyclic step geometry. Constraints on the 
foot velocity at touchdown enforce an impact invariant gait. Cyclic motions are guaranteed by using a linear projector on the parameter space of the optimization problem.

The infinitely dimensional trajectory optimization problem is discretized using B-Splines for parameterizing $\boldsymbol{y}(t)$ and $\boldsymbol{\alpha}(t)$. The cost function and the constraints are evaluated at discrete points during the motion.

Based on this optimization problem, we generate a set of trajectories for a given task space. In this example, the task space contains only the step length, but can also be used for larger task spaces as shown in [4]. The tasks can then be generalized using machine learning methods presented in [4], which introduces little error when predicting arbitrary steps in the whole range and produces a very compact representation of the optimal results.

In order to transition between two primitives, a noncyclic interpolated trajectory is generated by blending two step primitives in the configuration space [4]:

$$
\boldsymbol{y}(t)=\beta(t) \cdot \boldsymbol{y}_{1}(t)+(1-\beta(t)) \cdot \boldsymbol{y}_{2}(t)
$$

where $\boldsymbol{y}_{n}(t)$ are the two trajectories to be blended and $\beta(t)$ is a third order B-Spline which is twice continuously differentiable and thus ensures continuous second derivatives of the blended primitives. The latter is essential for generating a trajectory which results in continuous states at the motor side of each joint. The transition between $\boldsymbol{y}_{1}(t)$ and $\boldsymbol{y}_{2}(t)$ happens completely during the SSP.

In order to avoid transitions between primitives which lead to infeasible contact forces we propose the step planning algorithm presented in the following section.

\section{Step Planning}

In order to increase the robustness of robotic walking, step adaptation as a reaction to disturbances is essential. To combine the use of primitives with the reactivity of on-line control, two adaptation schemes are applied. The primary adaptation uses a predictive control scheme with a concentrated mass model to compute feasible next foot steps based on the current CoM position of the robot and the stepping tasks $\boldsymbol{k}$. For the selection of future steps we formulate the locomotion goal as a cost function:

$$
\begin{array}{r}
\Gamma=a_{0}\left(\hat{\boldsymbol{x}}_{\mathrm{c}, \mathrm{x}}-\boldsymbol{x}_{\mathrm{c}, \mathrm{x}}\left(t_{\mathrm{p}}\right)\right)+a_{1}\left(\dot{\hat{\boldsymbol{x}}}_{\mathrm{c}, \mathrm{x}}-\dot{\boldsymbol{x}}_{\mathrm{c}, \mathrm{x}}\left(t_{\mathrm{p}}\right)\right) \ldots \\
\ldots+a_{2} \int_{0}^{t_{\mathrm{p}}} \ddot{\boldsymbol{x}}_{\mathrm{c}, \mathrm{x}}^{2} \mathrm{~d} t
\end{array}
$$

where $\boldsymbol{x}_{\mathrm{c}, \mathrm{x}}(t)$ is the horizontal CoM position, $t_{\mathrm{p}}$ the prediction horizon, $\hat{\boldsymbol{x}}_{\mathrm{c}, \mathrm{x}}$ the desired terminal CoM position, and $a_{i}$ are constant weights. This cost function is minimized under the constraints for contact forces given by $(6)$.

The required contact forces for a motion during the single support phase can be computed by:

$$
\boldsymbol{W}_{\mathrm{C}}(t)=\operatorname{Adj}^{\mathrm{T}}\left(\boldsymbol{H}_{\mathrm{c}, \text { foot }}\left(\boldsymbol{x}_{\mathrm{c}}(t), \boldsymbol{k}\right)\right) \cdot m\left(\boldsymbol{g}+\ddot{\boldsymbol{x}}_{\mathrm{c}}(t)\right)
$$

where $\boldsymbol{H}_{\mathrm{c} \text {,foot }}\left(\boldsymbol{x}_{\mathrm{c}}(t), \boldsymbol{k}\right)$ is the transformation matrix from the CoM to the current contact frame and $\operatorname{Adj}(\cdot)$ is the adjoint mapping. When multiple contacts are active, the redundancy of the contact forces is resolved in the same manner as (9). Additionally, the maximum step length is constrained.

The CoM motion is parameterized using a B-Spline, which is fixed at the start to match the current state of the CoM of the robots. The positions of future contacts are parameterized by their relative position to the starting point. This forms a non-linear optimization problem which is solved on-line.

This predictive control scheme is executed once at each touchdown of the swing foot, and computes the trajectory for the next step. A feasible next step can be computed in at most $100 \mathrm{~ms}$ and the new trajectory can be commanded to the tracking controller when the next step starts.

From this computation, the next task $\boldsymbol{k}$ is extracted and the associated primitive is commanded to the robot. During the SSP the predictive control scheme is not used, hence it can only act against disturbances which have already occured.

During the execution of the step trajectory, the next stance is adjusted based on continuous feedback [18]. The Cartesian swing foot trajectory $\boldsymbol{H}_{\mathrm{DSP}, \mathrm{d}}$ is modified to $\boldsymbol{H}_{\mathrm{DSP}, \mathrm{d}}^{\prime}$ as follows:

$$
\boldsymbol{H}_{D S P, d}^{\prime}=\left[\begin{array}{cc}
\boldsymbol{I} & b_{0} \cdot \Delta x_{\mathrm{c}, \mathrm{x}}+b_{1} \cdot \Delta \dot{x}_{\mathrm{c}, \mathrm{x}} \\
0 & 1
\end{array}\right] \boldsymbol{H}_{D S P, d}
$$

where $\Delta x_{c, x}$ is the horizontal CoM position error and $b_{i}$ are constant weights. The two concepts complement each other as a better model with constraints is used in the predictive part and the heuristic part provides robustness against disturbances during the step.

\section{Tracking Control}

In order to follow the trajectory, we use a simplified version of our controller presented in [3], summarized below. The provided trajectories are handed to the controller as desired states and derivatives $\boldsymbol{y}, \dot{\boldsymbol{y}}$, and $\ddot{\boldsymbol{y}}$. The controller also receives the planned contact forces and the contact state.

In order to maintain the balance, the controller stabilizes the CoM position $\boldsymbol{x}_{\mathrm{c}}$ and the hip orientation $\boldsymbol{R}_{b}$ by generating a Cartesian compliance force $\boldsymbol{W}_{\mathrm{x}} \in \mathbb{R}^{3}$ consisting of the stiffness matrix $\boldsymbol{K}_{\mathrm{CoM}}$ and damping matrix $\boldsymbol{D}_{\mathrm{CoM}}$. Each foot can be operated in two different modes. In the first one, the end effectors are actively used for supporting the robot (balancing mode) by generating the required contact wrenches $\boldsymbol{W}_{\text {bal }}$. In the interaction mode, the pose of the foot is stabilized by a Cartesian compliance generating $\boldsymbol{W}_{\text {int }}$. Depending on the mode, the foot Jacobian is part of $\boldsymbol{J}_{\text {bal }}$ (balancing) or $\boldsymbol{J}_{\text {int }}$ (interaction). 
As detailed in [3], the desired error dynamics of the closed loop system can be defined as

$$
\boldsymbol{\Lambda}\left[\begin{array}{c}
\Delta \dot{\boldsymbol{v}}_{x} \\
\Delta \dot{\boldsymbol{v}}_{\text {bal }} \\
\Delta \dot{\boldsymbol{v}}_{\text {int }}
\end{array}\right]+\boldsymbol{\mu}\left[\begin{array}{c}
\Delta \boldsymbol{v}_{x} \\
\Delta \boldsymbol{v}_{\text {bal }} \\
\Delta \boldsymbol{v}_{\text {int }}
\end{array}\right]=\boldsymbol{W}_{\text {ext }}-\left[\begin{array}{c}
\boldsymbol{W}_{x} \\
\boldsymbol{W}_{\text {bal }} \\
\boldsymbol{W}_{\text {int }}
\end{array}\right]
$$

with the end effector velocities $\boldsymbol{v}_{\text {bal }}=\boldsymbol{J}_{\text {bal }}\left[\boldsymbol{v}_{\mathrm{x}}^{\mathrm{T}} \dot{\boldsymbol{q}}^{\mathrm{T}}\right]^{\mathrm{T}}$ and $\boldsymbol{v}_{\text {int }}=\boldsymbol{J}_{\text {int }}\left[\boldsymbol{v}_{\mathrm{x}}^{\mathrm{T}} \dot{\boldsymbol{q}}^{\mathrm{T}}\right]^{\mathrm{T}}$. The Cartesian inertia and Coriolis matrix are given by $\boldsymbol{\Lambda}$ and $\boldsymbol{\mu}$. Note that the translational velocity of the CoM and the rotational velocity of the hip are stacked into $\boldsymbol{v}_{\mathrm{x}}$. The $\Delta$ in (18) denotes the difference between the actual state of the system and the desired trajectory.

Comparing (18) with the dynamic model in task space coordinates (detailed in [3]) leads to the controller

$$
\left[\begin{array}{c}
\mathbf{0} \\
\boldsymbol{\tau}
\end{array}\right]=\left[\begin{array}{c}
m \boldsymbol{g}_{0}-\boldsymbol{W}_{\mathrm{x}} \\
\mathbf{0}
\end{array}\right]-\left[\begin{array}{cc}
\boldsymbol{J}_{\text {bal } \mathrm{u}}^{\mathrm{T}} & \boldsymbol{J}_{\text {int } \mathrm{T}, \mathrm{u}}^{\mathrm{T}} \\
\boldsymbol{J}_{\text {bal }, \mathrm{l}}^{\mathrm{T}} & \boldsymbol{J}_{\text {int }, \mathrm{l}}^{\mathrm{T}}
\end{array}\right]\left[\begin{array}{l}
\boldsymbol{W}_{\text {bal }} \\
\boldsymbol{W}_{\text {int }}
\end{array}\right]+\left[\begin{array}{c}
\boldsymbol{W}_{\mathrm{ff}} \\
\boldsymbol{\tau}_{\mathrm{ff}}
\end{array}\right]
$$

The Jacobian matrices are partitioned into $\boldsymbol{J}_{\mathrm{bal}, \mathrm{u}}$ (nonactuated), $\boldsymbol{J}_{\text {bal, },}$ (actuated) and $\boldsymbol{J}_{\text {int,u }}, \boldsymbol{J}_{\text {int,ll }}$. The last part represents feedforward terms similar to $\mathrm{PD}+$ control as detailed in [3].

The controller (19) can be computed within two steps: At first, we can use the first line to obtain $\boldsymbol{W}_{\text {bal }}$, which is then mapped via the second line to the control torques $\tau$. Note that the first line of (19) is underdetermined, which represents the force distribution problem of balancing on multiple end effectors. The redundancy is resolved via a quadratic optimization

$$
\min _{\boldsymbol{W}_{\text {bal }}}\left(\boldsymbol{W}_{\text {bal }}-\boldsymbol{W}_{\text {bal }}^{d}\right)^{T} \boldsymbol{Q}\left(\boldsymbol{W}_{\text {bal }}-\boldsymbol{W}_{\text {bal }}^{d}\right)
$$

with respect to the constraint

$$
\mathbf{0}=m \boldsymbol{g}_{0}-\boldsymbol{W}_{\mathrm{x}}-\boldsymbol{J}_{\text {bal }, \mathrm{u}}^{T} \boldsymbol{W}_{\text {bal }}-\boldsymbol{J}_{\text {int }, \mathrm{u}}^{T} \boldsymbol{W}_{\text {int }}+\boldsymbol{W}_{\mathrm{ff}}
$$

and to the contact model (6). The positive definite weight matrix is given by $\boldsymbol{Q}$. The balancing wrenches are then mapped to the control torque via the second line of (19):

$$
\boldsymbol{\tau}=-\boldsymbol{J}_{\text {bal }, 1}^{T} \boldsymbol{W}_{\text {bal }}-\boldsymbol{J}_{\text {int }, \mathrm{l}}^{T} \boldsymbol{W}_{\text {int }}+\boldsymbol{\tau}_{\text {fff }} .
$$

In order to increase efficiency of the tracking controller, we propose to replicate the intrinsic stiffness with the CoM impedance force $\boldsymbol{W}_{\mathrm{x}}$. This significantly lowers the required control effort, as the motor does not have to move to generate this behavior. In the SSP, the mechanism is constrained by

$$
\mathbf{0}=\left[\begin{array}{ll}
\boldsymbol{J}_{\text {foot,u }} & \boldsymbol{J}_{\text {foot, },}
\end{array}\right]\left[\begin{array}{c}
\boldsymbol{v}_{\mathrm{x}} \\
\dot{\boldsymbol{q}}
\end{array}\right] .
$$

where $\boldsymbol{J}_{\text {foot,u }}$ and $\boldsymbol{J}_{\text {foot,l }}$ denote the upper and lower part of the foot Jacobian. Solving for $\boldsymbol{v}_{\mathrm{x}}$ leads to

$$
\boldsymbol{v}_{\mathrm{x}}=\underbrace{-\boldsymbol{J}_{\text {foot }, u}^{-1} \boldsymbol{J}_{\text {foot, }, \mathrm{l}}}_{\boldsymbol{J}_{\text {constr, } \mathrm{CoM}}} \cdot \dot{\boldsymbol{q}}
$$

The resulting CoM stiffness can then be computed using:

$$
\boldsymbol{K}_{\mathrm{CoM}}=\left(\boldsymbol{J}_{\text {constr, } \mathrm{CoM}} \boldsymbol{K}^{-1} \boldsymbol{J}_{\text {constr }, \mathrm{CoM}}^{T}\right)^{-1}
$$

which is a local approximation of the real behavior as the state-dependent Jacobian introduces a more complex relation. For the double support phase $(D S P)$, both leg stiffnesses are in parallel:

$$
\boldsymbol{K}_{\mathrm{CoM}, \mathrm{DSP}}=\boldsymbol{K}_{\mathrm{CoM}, \mathrm{R}}+\boldsymbol{K}_{\mathrm{CoM}, \mathrm{L}}
$$

Extending this idea to the swing foot impedance, the required constraint Jacobian $\boldsymbol{J}_{\text {constr,SF }}$ is obtained by:

$$
\begin{aligned}
& \boldsymbol{J}_{\text {constr,SF }}=\left[\begin{array}{l}
\boldsymbol{J}_{\text {foot,u }}^{\mathrm{T}} \\
\boldsymbol{J}_{\text {foot }, 1}^{\mathrm{T}}
\end{array}\right]\left[\begin{array}{c}
\boldsymbol{J}_{\text {constr, } \mathrm{CoM}} \\
\boldsymbol{I}
\end{array}\right] \\
& \boldsymbol{K}_{\mathrm{SF}}=\left(\boldsymbol{J}_{\text {constr,SF }} \boldsymbol{K}^{-1} \boldsymbol{J}_{\text {constr,SF }}^{\mathrm{T}}\right)^{-1}
\end{aligned}
$$

In order to compute the diagonal damping matrix $\boldsymbol{D}$, which is required for the impedance control of the CoM and of the swing foot, the Cartesian mass must be determined first by

$$
\boldsymbol{M}_{\text {cart }}=\left(\boldsymbol{J}_{\text {constr }} \boldsymbol{M}^{-1} \boldsymbol{J}_{\text {constr }}^{\mathrm{T}}\right)^{-1} .
$$

based on the same constraint. Computing $\boldsymbol{D}_{\mathrm{CoM}}$, i.e. damping design for non-diagonal $\boldsymbol{K}_{\mathrm{F}}$ and $\boldsymbol{K}_{\mathrm{CoM}}$ can be done using the generalized Eigenvalue method.

\section{ViI. Series Elastic Actuator Control}

The series elastic actuator dynamics are given by (4). In this approach, we use a motor position control to remove especially the effects of the motor side friction. A cascaded control structure to realizes a motor position control:

$$
\begin{aligned}
\boldsymbol{\tau}_{m} & =K_{\mathrm{V}, \mathrm{P}} \cdot(\dot{\hat{\boldsymbol{\theta}}}-\dot{\boldsymbol{\theta}})+K_{\mathrm{V}, \mathrm{I}}(\hat{\boldsymbol{\theta}}-\boldsymbol{\theta})+\boldsymbol{B} \cdot \ddot{\boldsymbol{\theta}}_{\mathrm{d}}+\boldsymbol{\tau}_{\mathrm{d}} \\
\dot{\hat{\boldsymbol{\theta}}} & =K_{\mathrm{P}} \cdot\left(\boldsymbol{\theta}_{\mathrm{d}}-\boldsymbol{\theta}\right)+\dot{\boldsymbol{\theta}}_{\mathrm{d}}
\end{aligned}
$$

where $\hat{\boldsymbol{\theta}}$ is the input of the lower velocity level cascade and $\tau_{\mathrm{d}}$ is the desired link side torque. This torque is converted to a desired joint position with:

$$
\boldsymbol{\theta}_{\mathrm{d}}=\boldsymbol{K}^{-1} \boldsymbol{\tau}_{\mathrm{d}}+\boldsymbol{q} .
$$

\section{Results AND EXPERIMENTS}

To verify the effectiveness of this approach, we conducted several experiments on the bipedal robot CRunner, for which hardware details are available in [5]. C-Runner is a highly capable planar robot with series elastic actuators and a weight of $67 \mathrm{~kg}$. The base of the robot is free to move in the sagittal plane $(x, z$ and $\varphi$ ) while the other directions are constrained using a rotating boom which guides the robot. The robot has three actuated joints per leg. The flat feet allow the robot to balance without making a step.

For comparing this robot to other systems, it is most useful to look at the characteristic numbers regarding actuation of C-Runner: the motors have a maximum instantaneous motor torque of $400 \mathrm{Nm}$, a maximum motor velocity of $5.2 \mathrm{rad} / \mathrm{s}$, a projected motor inertia of $1.62 \mathrm{~kg} \cdot \mathrm{m}^{2}$, and a spring stiffness of $451 \mathrm{Nm} / \mathrm{rad}$, $560 \mathrm{Nm} / \mathrm{rad}$ and $423 \mathrm{Nm} / \mathrm{rad}$ for hip, knee and ankle joints respectively. The admissible link side torque is limited 


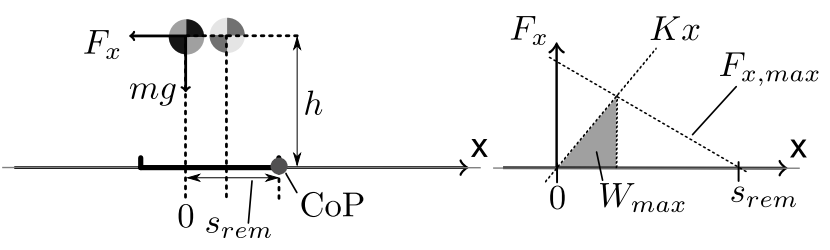

Fig. 4. $W_{\max }$ is the which is absorbed when a horizontal disturbance acts on the robot

to $200 \mathrm{Nm}$. The link side inertia in the hip joint for a typical configuration is $2.7 \mathrm{~kg} \cdot \mathrm{m}^{2}$. The motor position controller was parameterized with $K_{\mathrm{P}}=66.831 / \mathrm{s}$, $K_{\mathrm{V}, \mathrm{I}}=25.92^{\mathrm{Nm}} / \mathrm{rad}$ and $K_{\mathrm{V}, \mathrm{P}}=95.31^{\mathrm{Nm}} / \mathrm{rad} \cdot \mathrm{s}$.

The solution of the optimal control problem was described with 360 parameters for each step trajectory. The standard step timing with $0.6 \mathrm{~s}$ for the $S S P$ and $0.2 \mathrm{~s}$ for the DSP was used. The optimization solution is restricted to use only $60 \%$ of the maximum motor velocity and allowed ZMP range. Additionally, the motion was constrained to lift the swing foot by at least $20 \mathrm{~mm}$ during the forward swing.

The step planner has a prediction horizon of $2 \mathrm{~s}$ with a discretization of $0.1 \mathrm{~s}$ at which the cost function and the constraints are evaluated. The B-Spline parameterizing the CoM trajectory has 20 free parameters.

For the joint stiffness given above, the intrinsic Cartesian stiffness of the CoM in the SSP computes to:

$$
\boldsymbol{K}_{C o M}=\left[\begin{array}{ccc}
2220^{N} / \mathrm{m} & -2925^{N} / \mathrm{m} & -794^{\mathrm{N} / \mathrm{rad}} \\
-2925^{\mathrm{N}} / \mathrm{m} & 34205^{\mathrm{N}} / \mathrm{m} & -890^{\mathrm{N} / \mathrm{rad}} \\
-794^{\mathrm{Nm} / \mathrm{m}} & -890^{\mathrm{Nm} / \mathrm{m}} & 565^{\mathrm{Nm} / \mathrm{rad}}
\end{array}\right]
$$

these intrinsic stiffness values are not optimal for stability of the robot, given (6). It is possible to compute the optimal stiffness, starting from the maximum admissible horizontal instantaneous CoM force

$$
F_{\mathrm{x}, \max }=\frac{m g s_{\mathrm{rem}}}{h}
$$

where $m$ is the robots mass, $g$ the gravity, $s_{\text {rem }}$ the remaining support polygon in the direction the disturbance is pushing and the CoM height $h$. As illustrated in Fig. 4, the energy required $W_{\max }$ to push the robot over, given linear horizontal stiffness $K_{\mathrm{x}}$ is

$$
W_{\max }\left(K_{\mathrm{x}}\right)=\frac{K_{\mathrm{x}}}{2}\left[\frac{m g s_{\mathrm{rem}}}{h K_{\mathrm{x}}+m g}\right]^{2}
$$

with $s_{\text {rem }}$ the support polygon available at zero compliance force. Using $\partial W_{\max } / \partial K_{\mathrm{x}} \stackrel{!}{=} 0$ we can obtain the optimal spring stiffness $K_{\mathrm{x} \text {,opt }}$, for this particular robot this approach yields approximately $700 \mathrm{Nm} / \mathrm{m}$.

From our practical experience it is additionally good to decouple the $\mathrm{x}$ coordinate from $\mathrm{z}$ and $\phi$ :

$$
\boldsymbol{K}_{\mathrm{CoM}, \mathrm{var}}=\left[\begin{array}{ccc}
K_{\mathrm{x}, \mathrm{opt}} & 0 & 0 \\
0 & K_{\mathrm{z}} & K_{\mathrm{z} \phi} \\
0 & K_{\mathrm{z} \phi} & K_{\phi}
\end{array}\right] \quad \begin{array}{ccc}
2000 & \leq K_{\mathrm{z}} \leq 8000 \\
-1000 & \leq K_{\phi} \leq 1000 \\
\hline & &
\end{array}
$$

which leaves the variables $\left[K_{\mathrm{z}}, K_{\phi}, K_{\mathrm{z}, \phi}\right]$, bounded in the listed ranges. This desired Cartesian stiffness again can be realized intrinsically by introducing biarticulation, as mentioned in [5]. In the future this robot will be equipped with the following biarticulation possibilities:

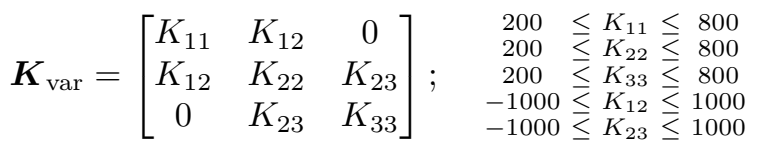

To find the best combination of SEA spring and biarticulation, we minimize the Frobenius norm over the difference between the projected intrinsic stiffness using (25) and $K_{\mathrm{CoM} \text {,var }}$. The resulting stiffness matrices are:

$$
\boldsymbol{K}_{\mathrm{opt}}=\left[\begin{array}{ccc}
200 & 141 & 0 \\
141 & 200 & 133 \\
0 & 133 & 234
\end{array}\right] ; \boldsymbol{K}_{\mathrm{CoM}, \mathrm{opt}, \mathrm{SSP}}=\left[\begin{array}{ccc}
837 & 45 & -404 \\
45 & 1097 & 237 \\
-404 & 237 & 282
\end{array}\right]
$$

for a typical configuration. The minimization was executed over a set of motion primitives, considering the different contacts with the environment. These stiffness values reduce the torque bandwidth of the robot.

However, there are effectively three points to take into account. (a) On the real system, using the current intrinsic stiffness as control stiffness leads to high gains as e.g. on the base position, which can be a problem in the presence of sensor noise. (b) $\boldsymbol{K}_{\mathrm{CoM}}$ and can be reduced by the contact stiffness depending on the configuration. (c) Because the force distribution problem respects (6), the use of the intrinsic system stiffness is not fully realized when the constraints are active.

\section{A. Experiment 1}

In the first experiment C-Runner walks a full circle, a distance of approx. $18 \mathrm{~m}$ while maintaining a velocity of $0.175 \mathrm{~m} / \mathrm{s}$. The cost of transport for walking is 0.13 for the link side, computed with $E=\int \dot{\boldsymbol{q}}^{\mathrm{T}} \cdot \boldsymbol{\tau} \mathrm{d} t$ as energy, 1.21 for the complete mechanical subsystem, computed with $E=\int \dot{\boldsymbol{\theta}}^{\mathrm{T}} \cdot \boldsymbol{\tau}_{\mathrm{m}} \mathrm{d} t$ thus including the motor side dynamics (4) and motor friction. The system power required to produce this gait is illustrated in Fig. 6. Fig. 5 shows that these high power peaks correlate with quick changes of link side torque. This plot especially shows how the relative degree of four between the joint position and the motor torque creates very demanding motor torque spikes. Accounting for losses in the power electronics, the electric cost of transport computes to 3.16 , which does not include power for sensing and computation. Careful design of the controller behavior during touchdown and lift-off is essential to reduce the power losses to a minimum. We found that a longer time span for switching from impedance to force control improves the behavior. However, there is very little time (200 ms) during the double support phase to realize these transitions.

Fig. 7 shows the tracking of the CoM during a phase of constant velocity. Reasonably good tracking is achieved in these phases. The measured foot positions are distorted by sensor noise in the base coordinates. During this experiment the predictive step adaptation generated the CoM trajectory depicted in Fig. 8, which illustrates the nominal case in which the step adaptation can reach the desired goal. 

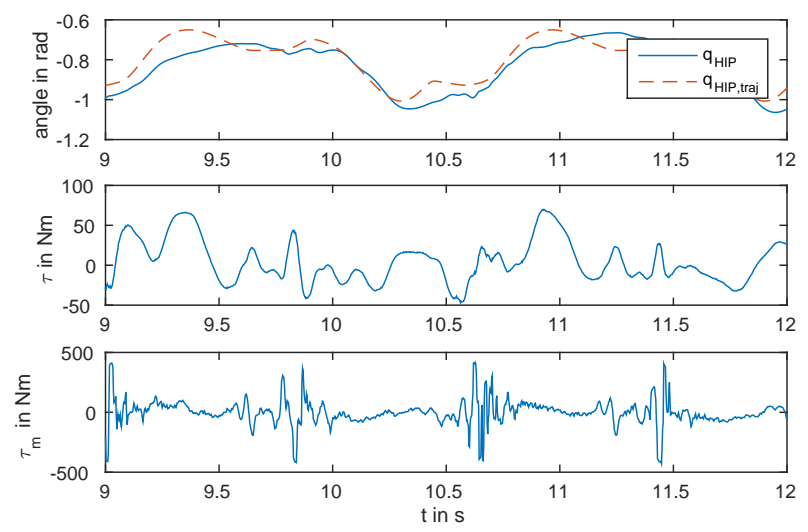

Fig. 5. Analysis of the hip joint during two steps. The first plot shows the planned (dashed) and the measured joint angle $q$ (solid). The second plot shows the joint torque $\tau$. The third plot shows the motor torque $\tau_{\mathrm{m}}$, which spikes on contact change to realize high torque dynamics.

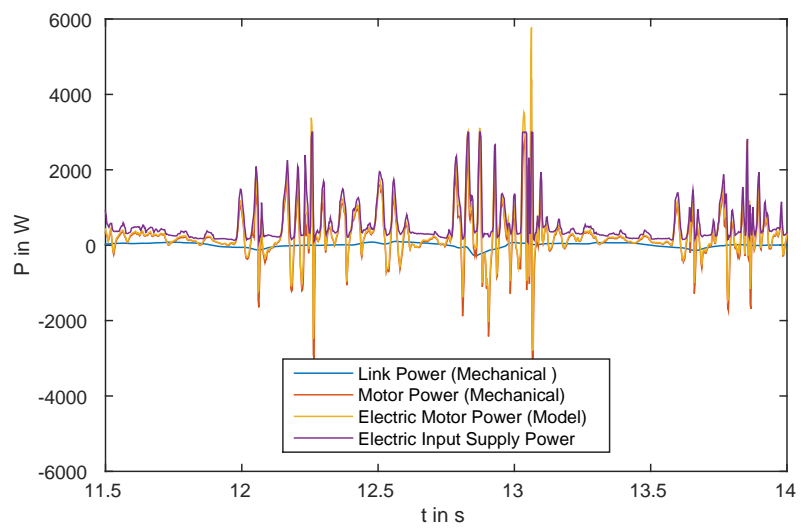

Fig. 6. System power over one step. Link side power $\dot{\boldsymbol{q}}^{\mathrm{T}} \cdot \boldsymbol{\tau}$, motor mechanical power $\dot{\boldsymbol{\theta}}^{\mathrm{T}} \cdot \boldsymbol{\tau}_{\mathrm{m}}$ and electric power drawn from supply $P_{\mathrm{IN}}$. Notice the power supply is limited at $3 \mathrm{~kW}$ but the capacitor bank is large enough to compensate this.

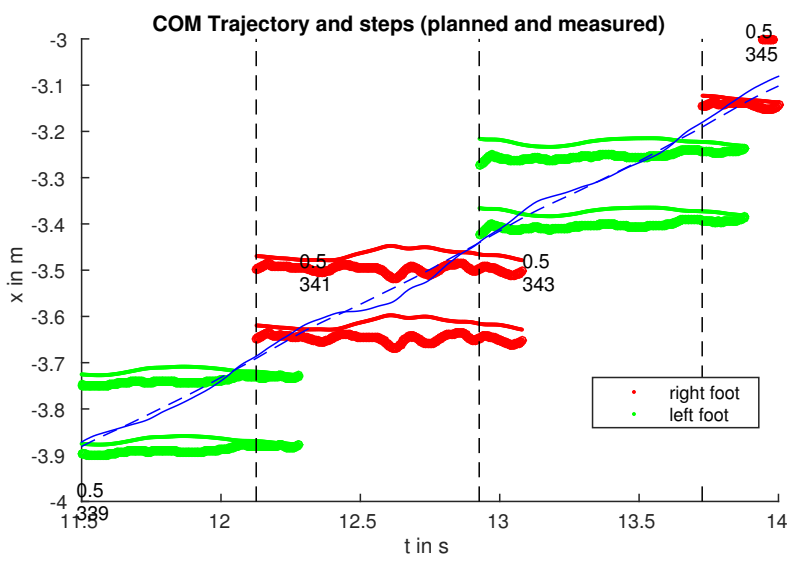

Fig. 7. CoM Tracking of foot position. The red/green thin lines mark the right/left planned foot positions, the thick lines the measured ones. The blue continuous line shows the measured CoM, the blue dashed line the desired. This plot shows a sequence of steps with a constant walking speed of $0.31 \mathrm{~m} / \mathrm{s}$.

\section{B. Experiment 2}

In the second experiment, the robot walks across an obstacle as shown in Fig. 1. The robot has no model of the $100 \mathrm{~mm}$ high obstacle which rises in two steps. Thus, the planned and the executed trajectory differ significantly. After traversing the front part of the obstacle, the obstacle tips over and introduces significant disturbance. This scenario shows the advantages of force controlled walking as unforeseen changes in the floor height create no problems. The step adaptation helps stabilizing the robot when the see-saw tips over and introduces a disturbance.

\section{Conclusions}

Increasing walking velocity uses more of the system capabilities leaving less room for additional control torque to be realized. This is especially the case as the control torque often contains high frequency components which require significant motor torque to be successfully tracked.

As we use a cascade control architecture containing a balancing and a motor position controller (see Fig.2), it is essential that $\boldsymbol{\tau}_{\mathrm{d}}$ given by the balancing controller can be tracked. For this, the motor position controller has to have significantly faster settling time than the balancing controller. However, this is not always possible because of the limitations of the actuator speed $\dot{\boldsymbol{\theta}}$ and motor torque $\boldsymbol{\tau}_{\mathrm{m}}$. This results in limited dynamics of $\boldsymbol{\tau}$ which cause a loss of efficiency in the control concept. There are three ways to remedy this problem:

- Acceptance of the system behavior and replicating it in the tracking controller. However not all aspects of the current intrinsic behavior are desired.

- Adaptation of the joint stiffness $\boldsymbol{K}$ to realize the desired behavior. While this will improve the regulation behavior, it significantly reduces the ability to produce quick link motions, which limits e.g. the step adaptation capabilities. Using the stiffness $\boldsymbol{K}_{\text {opt }}$, it is not possible with the trajectory generation to generate primitives when keeping the step timing and step length, only much slower walking is feasible.

- Changing the SEA unit such that it can realize higher dynamics in $\boldsymbol{\tau}$ at the link side. For example, this could be done by adding a damper [19] to the coupling. This reduces the relative degree between $\boldsymbol{\tau}$ and $\boldsymbol{\tau}_{\mathrm{m}}$, enabling high dynamics on the link side as effect of the motor-side limitations are differently mapped to the torque dynamics. This also allows to implement the damping, normally implemented by the controller, intrinsically.

We also evaluate trajectories generated for an equivalent rigid body robot, but it is not possible to generate smooth walking with a velocity above $0.1 \mathrm{~m} / \mathrm{s}$. This can be attributed to nominal torque profiles required by the rigid body trajectories which cannot be generated by the SEAs. 


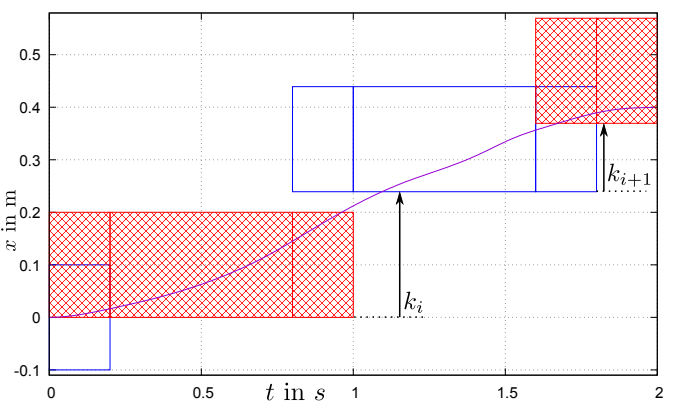

Fig. 8. Step generation at start of walking. The right foot outline is displayed as red hatched area, the left foot outline is displayed in blue. The $\mathrm{x}$ axis shows the time over which the contacts are active, the blue line shows the feasible CoM trajectory.

To change the natural behavior as little as possible, we used a sub-critical damping of $\xi=0.3$ with some additional damping provided by the friction inherent to the mechanism.

There are no limitations which would restrict this method from producing similar gait for straight walking on a non-planar robot.

The cost of transport in comparison to robots like DURUS is still high, however DURUS is only compliant in the normal direction of the contact. C-Runner in comparison provides compliance in all joints. This compliance comes at a cost, but also provides more robustness e.g. when falling. While exploiting the natural dynamics is possible in this theoretical framework, the system design must still be improved to realize improvements regarding the cost of transport.

The essential dynamics of walking are covered in three parts of this work. Trajectory planning, step planning, and balancing all mainly deal with the CoM dynamics and contact points. We keep the mathematical formulations as similar as possible to avoid the use of proxyconstraints to enforce restrictions of underlying control methods in the planning phase. Only the values of the contact force constraints differs on the levels, the planning always being more conservative than the control.

\section{Future Work}

The trajectory generation process can be extended to human like walking, which includes underactuation. Also the framework is general enough but requires moderate extension to realize running.

On the system design level, the stiffness of the robot is found to be too high for unmodified use as control stiffness. However, lower stiffness alone decreases the torque control bandwidth. We will investigate the possibilities of different coupling mechanisms, such as intrinsic damping, to maintain bandwidth and lower stiffness at the same time.

\section{REFERENCES}

[1] D. Lakatos, A. Albu-Schäffer, C. Rode, and F. Loeffl, "Dynamic bipedal walking by controlling only the equilibrium of intrinsic elasticities," in 2016 IEEE-RAS 16th International Conference on Humanoid Robots (Humanoids). IEEE, 2016, pp. $1282-1289$.
[2] A. Werner, R. Lampariello, and C. Ott, "Trajectory optimization for walking robots with series elastic actuators," in 53rd Conference on Decision and Control (CDC), 2014, pp. 29642970.

[3] B. Henze, M. A. Roa, and C. Ott, "Passivity-based whole-body balancing for torque-controlled humanoid robots in multicontact scenarios," The International Journal of Robotics Research, vol. 35, no. 12, pp. 1522-1543, 2016.

[4] A. Werner, D. Trautmann, D. Lee, and R. Lampariello, "Generalization of optimal motion trajectories for bipedal walking," in 2015 IEEE/RSJ International Conference on Intelligent Robots and Systems (IROS). IEEE, 2015, pp. 1571-1577.

[5] F. Loeffl, A. Werner, D. Lakatos, J. Reinecke, S. Wolf, R. Burger, T. Gumpert, F. Schmidt, C. Ott, M. Grebenstein, et al., "The dlr c-runner: Concept, design and experiments," in 2016 IEEE-RAS 16th International Conference on Humanoid Robots (Humanoids). IEEE, 2016, pp. 758-765.

[6] J. E. Pratt and B. T. Krupp, "Series elastic actuators for legged robots," in Defense and Security. International Society for Optics and Photonics, 2004, pp. 135-144.

[7] J. Pratt, T. Koolen, T. De Boer, J. Rebula, S. Cotton, J. Carff, M. Johnson, and P. Neuhaus, "Capturability-based analysis and control of legged locomotion, part 2: Application to $\mathrm{m} 2 \mathrm{v} 2$, a lower-body humanoid," The International Journal of Robotics Research, vol. 31, no. 10, pp. 1117-1133, 2012.

[8] Z. Li, N. G. Tsagarakis, and D. G. Caldwell, "A passivity based admittance control for stabilizing the compliant humanoid coman," in 2012 12th IEEE-RAS International Conference on Humanoid Robots (Humanoids). IEEE, 2012, pp. 43-49.

[9] B. G. Buss, A. Ramezani, K. A. Hamed, B. A. Griffin, K. S. Galloway, and J. W. Grizzle, "Preliminary walking experiments with underactuated 3d bipedal robot marlo," in 2014 IEEE/RSJ International Conference on Intelligent Robots and Systems (IROS). IEEE, 2014, pp. 2529-2536.

[10] K. A. Hamed and J. W. Grizzle, "Event-based stabilization of periodic orbits for underactuated 3-d bipedal robots with left-right symmetry," IEEE Transactions on Robotics, vol. 30, no. 2, pp. 365-381, 2014.

[11] J. W. Grizzle, J. Hurst, B. Morris, H.-W. Park, and K. Sreenath, "Mabel, a new robotic bipedal walker and runner," in American Control Conference, 2009 (ACC). IEEE, 2009, pp. 2030-2036.

[12] E. R. Westervelt, J. W. Grizzle, and D. E. Koditschek, "Hybrid zero dynamics of planar biped walkers," IEEE Transactions on Automatic Control, vol. 48, no. 1, pp. 42-56, 2003.

[13] E. R. Westervelt, J. W. Grizzle, C. Chevallereau, J. H. Choi, and B. Morris, Feedback control of dynamic bipedal robot locomotion. $\quad$ CRC press, 2007, vol. 28.

[14] C. Chevallereau, J. W. Grizzle, and C.-L. Shih, "Asymptotically stable walking of a five-link underactuated 3-d bipedal robot," IEEE Transactions on Robotics, vol. 25, no. 1, pp. 3750,2009

[15] K. Sreenath, H.-W. Park, I. Poulakakis, and J. W. Grizzle, "A compliant hybrid zero dynamics controller for stable, efficient and fast bipedal walking on mabel," The International Journal of Robotics Research, vol. 30, no. 9, pp. 1170-1193, 2011.

[16] A. Hereid, E. A. Cousineau, C. M. Hubicki, and A. D. Ames, "3d dynamic walking with underactuated humanoid robots: A direct collocation framework for optimizing hybrid zero dynamics," in 2016 IEEE International Conference on Robotics and Automation (ICRA). IEEE, 2016, pp. 1447-1454.

[17] A. Werner, R. Lampariello, and C. Ott, "Optimization-based generation and experimental validation of optimal walking trajectories for biped robots," in International Conference on Intelligent Robots and Systems (IROS), 2012, pp. 4373-4379.

[18] J. K. Hodgins and M. Raibert, "Adjusting step length for rough terrain locomotion," IEEE Transactions on Robotics and Automation, vol. 7, no. 3, pp. 289-298, 1991.

[19] M. J. Kim, A. Werner, F. C. Loeffl, and C. Ott, "Joint torque control capability of series elastic actuators with physical damping," in 2017 IEEE International Conference on Robotics and Automation (ICRA). IEEE, 2017. 